\title{
Admission to NICU in air is more likely if nasal High Flow is used for stabilisation in preterm babies compared to face mask CPAP
}

\author{
EDIT MOLNAR, NICOLA HOLLAND, PETER REYNOLDS \\ Neonatal Intensive Care Unit, St. Peter's Hospital, Surrey UK \\ Corresponding Author \\ Peter Reynolds \\ Neonatal Intensive Care Unit \\ St. Peter's Hospital, Ashford and St. Peter's Hospitals NHS Foundation Trust \\ Guildford Road, Surrey, UK \\ E-mail:peter.reynolds@asph.nhs.uk
}

\section{ABSTRACT}

Objective. To examine the success of stabilisation and the short term outcomes from the routine use of nasal high flow (nHF) on an unselected cohort of babies in the delivery room (DR).

Design. Retrospective single-centre study Setting. Single-centre neonatal intensive care unit (NICU)

Patients. Infants born at $<32$ weeks gestation

Interventions. Stabilisation and transfer to NICU of an unselected cohort of babies using $\mathrm{nHF}$

Main outcome measures. Success of stabilisation defined by successful transfer on $\mathrm{nHF}$ and clinical measures of stability at admission to NICU, including oxygen requirement, admission temperature, surfactant requirement, short term outcomes and whether infants were sustained on nHF by 72 hours of age.

Results. There were 133 eligible babies. 54 were commenced on $\mathrm{nHF}$ in the DR (Group A), 47 were stabilised by face mask CPAP (continuous positive airway pressure) (Group B), 26 were intubated (Group C); 6 required only minimal respiratory support (Group D). Median maturity varied between the groups (Group A $27+5$ weeks, Group B 30 weeks, Group C $26+2$ weeks, Group D 31+5). $72 \%$ of Group A and $75 \%$ of Group B remained on nHF for 72 hours $(\mathrm{P}=0.82)$. Fewer babies received surfactant in Group A versus Group B (29\% vs 35\%; P=0.67), however groups were not matched for maturity differences and Group A were significantly less mature and of lower birthweight (both $\mathrm{P}<0.001)$. Group A were significantly more likely to be in air at admission than Group $\mathrm{B}(\mathrm{P}=0.03)$.

Conclusion. Preterm babies can be successfully stabilised and sustained on nHF.
The use of nHF for immediate stabilisation appears to be effective and, in this study, led to significantly more babies being in air on admission to the NICU compared to face mask CPAP stabilisation.

Key words: nasal High Flow cannula, delivery room, stabilisation, premature

\section{INTRODUCTION}

The majority of babies born prematurely require stabilisation, not resuscitation, in the Delivery Room (DR). Whilst the latest guidance supports the use of nasal Continuous Positive Airway Pressure (CPAP), $(1,2)$ with evidence of improved outcomes compared with routine invasive respiratory support, $(3,4)$ we recently demonstrated the feasibility of the use of nasal high flow (nHF) in the stabilisation of premature babies ( 23 to $29+6$ weeks gestation) in the delivery room prior to transfer to Neonatal Intensive Care Unit (NICU). (5)

Although several large studies have previously demonstrated that nHF is generally as effective as nCPAP for postextubation support, (6-8) there remains a paucity of literature on the use of $\mathrm{nHF}$ in the DR.

Our previous study showed that stabilisation on nHF reduced the rate of DR intubation and surfactant administration compared to our prior practice. However our work was biased due to the requirement for consent to be obtained prior to delivery, which probably selected a "healthier" population of babies due to the time available for antenatal preparation with steroids, magnesium and interventions for optimal maternal health and delivery. It is thus arguably more relevant to monitor interventions in a "real-world" setting. Here we present data on a subsequent unselected cohort of preterm babies who underwent stabilisation in the Delivery Room, with nHF where possible.

\section{MATERIALS AND METHODS}

\section{Study design}

This was a retrospective observational study to examine the short term outcomes from the routine use of nHF to stabilise an unselected cohort of babies born before 32 weeks gestation in the DR. We also wanted to see whether there were differences between using $\mathrm{nHF}$ and face mask CPAP for stabilisation, and results were compared using Fisher's exact test and Student's t-test as appropriate.

\section{Setting}

We collected data for babies born between 23 and 31+6 weeks at St Peter's Hospital, Chertsey, Surrey, UK between April 2015 and October 2016. We recorded the infant's gestational age, birthweight, whether babies required $\mathrm{nHF}$, nCPAP or intubation in the DR, admission $\mathrm{FiO}_{2}$ and temperature, need for surfactant and inotropes. We gathered information about pneumothorax, pulmonary haemorrhage, severe IVH (Intraventricular haemorrhage) (grade IIIIV) and whether nHF was sustained for 72 hours. Stabilisation of babies using $\mathrm{nHF}$ is our routine practice, therefore parental consent was not sought.

We used the same mobile nHF apparatus we previously built (figure 1). This device can be taken to each delivery and takes about 5 minutes to reach $37^{\circ} \mathrm{C}$. We followed the same clinical protocol for stabilisation as we did in our pilot study (figure 2). 


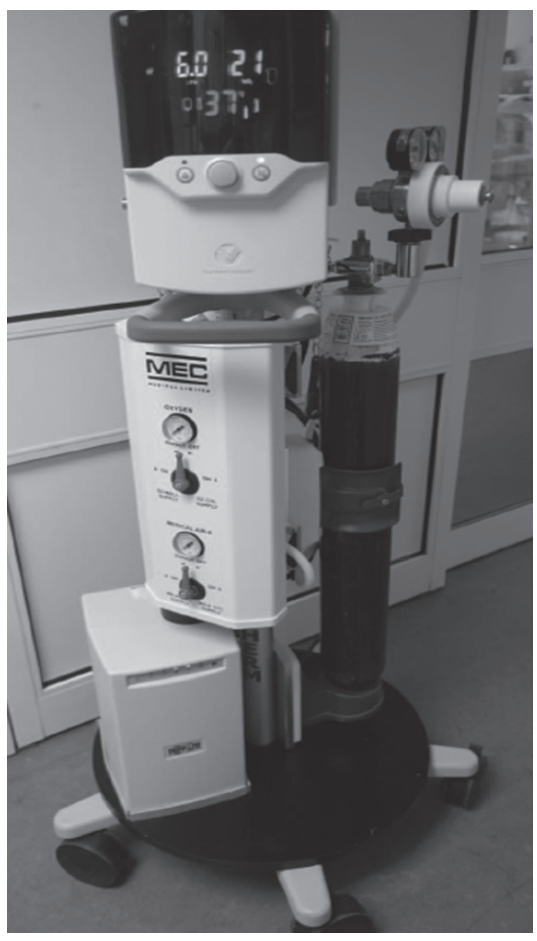

Figure 1. The mobile $n H F$ unit

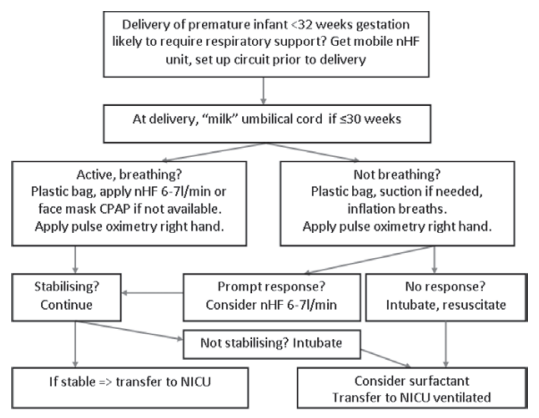

Figure 2. Protocol for use of nasal High

Flow in babies $<32$ weeks gestation

CPAP, continuous positive airway pressure; nHF, nasal High Flow ; NICU, neonatal intensive care unit.

\section{RESULTS}

\section{Participants}

A total of 133 eligible babies were admitted to NICU during the study period. Mean birth weight was 1080g (median 1014, mode 1100, range 340-2140, CI 1014-1145, SD 383) and mean gestational age $28+2$ weeks (median $28+1$, mode $27+2$, range $23+1-31+6$, CI $28+0-28+5$, SD 2+1).

\section{Outcomes}

Group A consisted of babies commenced on $\mathrm{nHF}$ in the DR. Group B consisted of babies stabilised using face mask CPAP in the DR. Group C were babies who required intubation and Group D were babies who required minimal or no additional stabilisation. Results are presented as median values.

There were 54 (41\%) babies with median gestation $27+5$ weeks and birthweight $935 \mathrm{~g}$ in Group A. Group B consisted of $48(36 \%)$ babies with median gestation of $30+0$ weeks and birthweight $1267 \mathrm{~g}$. Group C contained 25 infants (19\%), ranging from $23+1$ to $30+4$ weeks gestation (median 26+2) and birthweight $730 \mathrm{~g}$ (range 340-1537g). Group D consisted of 6 babies with a median gestational age of $31+5$ weeks and median birthweight $1695 \mathrm{~g}$ (range 1340-2140g).

In Group A, the median admission temperature was $36.8^{\circ} \mathrm{C}$ and $\mathrm{FiO}_{2}$ on admission was 0.25 , with a mode value of 0.21 in 22 (43\%) of the 51 babies in whom this was recorded. 16 (29\%) needed surfactant and $15(28 \%)$ required intubation $(12(80 \%)$ of those were given surfactant), and 4 (7\%) babies received inotropes during the first 72 hours. 39 (72\%) were sustained on nHF during the first 72 hours, 4 (10\%) of them required surfactant given by LISA (Less Invasive Surfactant Administration) procedure, and 4 (7\%) received inotropes. The 4 babies requiring surfactant but sustained on $\mathrm{nHF}$ had a median gestation of $27+2$ weeks (CI 24+0-29+4) and birth weight of 758g (CI 423-1185), and one of these had a Grade III/IV IVH, but no other complication was noted. 3 (6\%) babies in Group A had IVH Grade III/IV and no baby had a pulmonary haemorrhage. Two (4\%) babies developed pneumothoraces. The least mature baby in Group A was 24+1 weeks gestation and remained on nHF by 72 hours of age.

In Group B $(n=47)$ the median admission temperature was $36.9^{\circ} \mathrm{C}$, and $\mathrm{FiO}_{2}$ on admission was 0.3 . Ten (22\%) of those recorded $(n=45)$ were in air on admission to NICU. Babies were started on nHF on admission to NICU as per routine practice. $17(36 \%)$ required surfactant. 36 (77\%) remained on HF for at least 72 hours, 8 (22\%) of those received surfactant by the LISA procedure. The 8 babies requiring surfactant but sustained on nHF had a median gestation of $29+5$ weeks (CI $27+0$ $30+5$ ) and birth weight of $1157 \mathrm{~g}$ (CI 9461361). The smallest baby was $24+3$ weeks gestation with birth weight $780 \mathrm{~g} .12$ babies $(26 \%)$ required intubation, 9 of those (75\%) received surfactant. 1 (2\%) baby developed a pneumothorax and received inotropes. There were no babies with pulmonary haemorrhage or IVH grade III/IV.
In Group $C(n=26)$ the median admission temperature was $36.9^{\circ} \mathrm{C}$ (range $36^{\circ} \mathrm{C}$ - 38.3 ${ }^{\circ} \mathrm{C}$; CI 36.6-37.2;), with $\mathrm{FiO}_{2}$ on admission $0.3(\mathrm{n}=17$; range $21 \%-100 \%$; CI $0.23-0.49$ ) with 7 (27\%) babies in room air. 5 (19\%) received inotropes, 2 (8\%) babies developed a pneumothorax, $2(8 \%)$ babies had Grade III or IV IVH and 1 (4\%) baby suffered a pulmonary haemorrhage. 24 (92\%) babies received surfactant, 3 (12\%) were extubated on to HF on the unit and sustained on HF by 72 hours of life, all of them receiving surfactant before extubation.

Two (1.5\%) babies required facial oxygen only in the DR, 1 of them was subsequently intubated on the unit. Four babies (3\%) were breathing in air unsupported. None of these babies required surfactant, inotropic support or had pneumothorax, pulmonary haemorrhage, IVH grade III/IV.

Table 1 shows the analysis of Groups A and B. There were significant differences between the groups in the Gestational Ages and Birthweights, with Group B containing more mature and heavier babies. Babies in Group A were significantly more likely to be in room air by the time of admission to NICU $(\mathrm{P}=0.03)$.

\section{DISCUSSION}

In our previous pilot study (5) we showed that $60 \%$ of preterm babies could be sustained on nHF for at least the first 72 hours. In this work we demonstrated that $72 \%$ of babies who received $\mathrm{nHF}$ in the DR were sustained on nHF for 72 hours, so there has been an apparent improvement despite the population being unselected, although this might simply be due to small study numbers. Our antenatal steroid administration rates are still approximately $90 \%$, which probably accounts for the relatively good respiratory status of the preterm babies in the study.

In this study, we show that significantly more babies were in room air by the time of admission to NICU if stabilised on nHF, rather than facemask CPAP. This implies a high level of clinical stability, which is in line with our experiences. However there are no differences between Groups A and $B$ between the stabilisation modes in terms of sustained non-invasive ventilation.

The stabilisation of babies using non-invasive techniques also reduces surfactant use compared to historical practices. Our data also shows that $43 \%$ of all babies $<32$ weeks required surfactant. This compares favourably with our previous study where $48 \%$ of babies were given surfactant. In this 
Table 1. Comparison of babies stabilised in the DR with either $n$ HF or mask CPAP (continuous

\begin{tabular}{|c|c|c|c|}
\hline & Group A (nHF) & Group B (mask CPAP) & \\
\hline Number of babies & 54 & 47 & \\
\hline Gestational Age (weeks) & $\begin{array}{l}\text { Median: } 27+5 \\
\text { Range: } 24+1-31+5 \\
(\text { CI } 27+3-28+3 ; \text { SD 1+5) }\end{array}$ & $\begin{array}{l}\text { Median: } 30+0 \\
\text { Range: } 24+3-31+6 \\
(\text { CI } 29+0-30+0 ; \text { SD 1+5) }\end{array}$ & $\mathrm{P}=<0.001^{*}$ \\
\hline Birth weight (grams) & $\begin{array}{l}\text { Median: } 935 \\
\text { Range: } 465-1680 \\
\text { (CI 883-1046; SD 299) }\end{array}$ & $\begin{array}{l}\text { Median: } 1267 \\
\text { Range: } 660-2088 \\
\text { (CI 1185-1379; SD 333) }\end{array}$ & $\mathrm{P}=<0.001^{\star}$ \\
\hline nHF sustained for $72 \mathrm{hrs}$ & $39(72 \%)$ & $36(77 \%)$ & $\mathrm{P}=0.66^{* *}$ \\
\hline Surfactant given & $16(30 \%)$ & $17(36 \%)$ & $\mathrm{P}=0.53^{\star *}$ \\
\hline Inotropes given & $4(7 \%)$ & $1(2 \%)$ & $\mathrm{P}=0.37^{\star *}$ \\
\hline Pneumothorax & $2(4 \%)$ & $1(2 \%)$ & $\mathrm{P}=1.0^{* *}$ \\
\hline Pulmonary Haemorrhage & 0 & 0 & $\mathrm{P}=1.0^{* *}$ \\
\hline IVH Grade III/IV & $3(6 \%)$ & 0 & $\mathrm{P}=0.25^{\star *}$ \\
\hline Admission Temperature $\left({ }^{\circ} \mathrm{C}\right)$ & $\begin{array}{l}\text { Median: } 36.8 \\
\text { Range: } 34.5-38 \\
(\mathrm{CI} 36.7-37.1 \text {; SD 0.59) } \\
(\mathrm{n}=54)\end{array}$ & $\begin{array}{l}\text { Median: } 36.9 \\
\text { Range: } 36-38 \\
(\mathrm{CI} 36.8-37.1 ; \text { SD } 0.44) \\
(\mathrm{n}=47)\end{array}$ & $\mathrm{P}=0.8^{\star}$ \\
\hline Admission $\mathrm{FiO} 2$ & $\begin{array}{l}\text { Median } 0.25 \\
\text { Range: } 0.21-0.7 \\
(\mathrm{CI} 0.26-0.35 ; \text { SD } 0.15) \\
(\mathrm{n}=51)\end{array}$ & $\begin{array}{l}\text { Median } 0.30 \\
\text { Range } 0.21-0.6 \\
(\mathrm{CI} 0.28-0.34 \text {; SD } 0.08) \\
(\mathrm{n}=45)\end{array}$ & $\mathrm{P}=0.51^{*}$ \\
\hline Babies in FiO2 0.21 on admission to NICU & $22(\mathrm{n}=51 ; 43 \%)$ & $10(n=45 ; 22 \%)$ & $\mathrm{P}=0.03^{\star *}$ \\
\hline
\end{tabular}

study, $30 \%$ of babies received surfactant in Group A compared to $36 \%$ in Group B. This was not a significant difference but did not account for the significant differences in maturity and weight between the 2 groups. Since group B was, on average, 11 days more mature and 315 grams heavier at birth, this may mask important differences in sustained nHF success and surfactant use, and a controlled study is required to investigate that. Unsurprisingly the smallest and least mature babies required intubation in the DR. Almost all of the babies intubated required surfactant administration (92\%).

The rates of complications are comparable with previous stabilisation studies using nCPAP. We fully acknowledge the limitations of this work, which is a retrospective evaluation in a single centre with considerable expertise in managing preterm babies on nHF, and with excellent back- ground rates of antenatal steroid use. However we believe that the stability at admission of the majority of babies, the significant numbers of babies in air by the time of admission to NICU, the high proportions sustained on $\mathrm{nHF}$ for at least 72 hours, and the trend towards lower surfactant use should be encouraging for clinical teams who wish to consider alternative stabilisation strategies. We speculate that the use of $\mathrm{nHF}$ may reduce the need for oxygen due to the positive effects of inhaling warmed, humidified gas in reducing work of breathing compared to the cold dry gas used for face mask stabilisation.

\section{CONCLUSION}

We have shown that, under non-study conditions, it is feasible to stabilise and sustain babies using $\mathrm{nHF}$ in the DR. This practice led, in this study, to significantly more babies being in air by the time of admission, and reduced surfactant use compared to our historical practice. Our work was performed in a single centre with considerable experience in the use of nHF in babies, using a single device (Vapotherm Precision Flow) and our experiences and results cannot be automatically extrapolated to other situations or devices. However nHF in the DR appears to offer effective stabilisation for the majority of premature babies and remains our standard practice.

Acknowledgements

Thanks to the staff at St. Peter's Hospital NICU for their accurate completion of data which allowed this analysis to be carried out.

\section{REFERENCES}

1. Wyllie J, Bruinenberg J, Roehr CC, Rüdiger M, Trevisanuto D, Urlesberger B. European resuscitation council guidelines for resuscitation 2015: section 7. Resuscitation and support of transition of babies at birth. Resuscitation 2015;95:249-63.

2. Sweet DG, Carnielli V, Greisen G, Hallman M, Ozek E, Plavka R, et al. European Consensus Guidelines on the Management of Res- 
piratory Distress Syndrome - 2016 Update. Neonatology 2017;111(2):107-25.

3. Finer NN, Carlo WA, Walsh MC, Rich W, Gantz MG, Laptook AR, et al. SUPPORT Study Group of the Eunice Kennedy Shriver NICHD Neonatal Research Network. Early CPAP versus surfactant in extremely preterm infants. N Engl J Med 2010;362:1970-9.

4. Schmölzer GM, Kumar M, Pichler G, Aziz K, O’Reilly M, Cheung PY. Non-invasive versus invasive respiratory support in preterm infants at birth: systematic review and meta-analysis. BMJ 2013;347:f5980.

5. Reynolds P, Leontiadi S, Lawson T, Otunla T, Ejiwumi O, Holland N. Stabilisation of premature infants in the DR with nasal high flow. Arch Dis Child Fetal Neonatal Ed 2016;101:F284-7.

6. Yoder BA, Stoddard RA, Li M, King J, Dirnberger DR, Abbasi S. Heated humidified high-flow nasal cannula versus nasal CPAP for respiratory support in neonates. Pediatrics 2013;131:e1482-90.

7. Collins CL, Holberton JR, Barfield C, Davis PG. A randomised controlled trial to compare heated humidified high-flow nasal cannulae with nasal continuous positive airway pressure postextubation in premature infants. J Pediatr 2013;162:949-54.e1.

8. Manley BJ, Owen LS, Doyle LW, Andersen CC, Cartwright DW, Pritchard MA, et al. High-flow nasal cannulae in very preterm infants after extubation. N Engl J Med 2013;369:1425-33. 